\title{
A Review: The Survey of the Effects of Light on Weed Recognition
}

\author{
Zhengqiang Chen ${ }^{1}$ and Zhaomin $\mathrm{Ma}^{1}$ \\ ${ }^{1}$ College of Electric and Information Engineering, Guangxi University of Science and Technology, Liuzhou, Guangxi, China
}

\begin{abstract}
Weeding is one of the important tasks in agricultural field management. With the development of society and information technology, automatic weeding has become a developing trend. The automatic recognition of weeds based visual is the important step. In this paper, the effects of lighting on green identification and weed identification algorithms are summarized. In order to improve the accuracy and stability of the identification of crops and weeds, some further worth problems in the study are also put forward. This will help further research on automatic weeding.
\end{abstract}

\section{Introduction}

It is inevitable to associate with weeds in crop growth. Weeding is always one of the important tasks in agricultural field management. Automatic weed control has become a development trend with the development of society, information technology and other aspects. Automatic weeding can improve the cost-effectiveness and efficiency of agriculture. In particular, accurate recognition of weeds can improve the efficiency of herbicides. And accurate recognition of weeds is also one of the effective strategies to improve the sustainable development of agriculture. Automatic recognition of weeds is an indispensable step in both automatic spraying and automatic mechanical weeding of herbicides.

At present, the weed recognition method based on machine vision has been widely adopted with the research of image processing technology, information processing technology and pattern recognition algorithm. The original input of the recognition system comes from images or videos taken in fixed conditions. The images are affected by the light in an outdoor farming environment. Even agricultural images of the same target taken in succession vary greatly in image quality due to the instantaneous change of weather. The parameters of algorithm and sample sets are affected inordinately. The series of studies on the effects of light on weed recognition has been conducted.

\section{The effects of light on weed recognition}

Identification of green vegetation (crop and weed) and automatic identification of between weed and crop (weed identification) are two key steps in visual weed recognition. Images are the foundation of each step. At present, the hardware format of digital image is mostly RGB. The values of $\mathrm{R}$ (red component), $\mathrm{G}$ (green component) and B (blue component) of each pixel are reflected by different light environment when the images of the same agricultural target are taken even continuously. Sudden shadows, strong or weak sunlight, different weather, and so on, can have an impact throughout the process of weed recognition. The effect of light on algorithms of identification of green vegetation and automatic identification of between weed and crop is an important concern in this field.

\subsection{The effects of light on green vegetation identification}

The Green vegetation identification is the basis of weed identification, which is the process of extracting green vegetation target from soil and other background. This process is not only the basis of weed identification but also the basic step of acquiring other agricultural field target information based on visual (such as extraction of crop row, determination of field navigation line). The image of crops in the field is the input of green vegetation identification and image quality will be affected by the uncontrollable lighting environment in outdoor fields. The threshold segmentation method based on excess green vegetation index $(\mathrm{ExG})$ is often used in green vegetation identification traditionally. Then the segmentation threshold $\mathrm{T}$ is different under different lighting environment. The researchers did more studies in the following aspects to improve the effect of different light environments on the segmentation results.

Firstly, the development of the composite application of the capturing system and its components are studied. In [1] two different cameras were employed for capturing sugarcane images. Camera one was an intex IT-105 web camera (Cam1) and it was used for capturing inter-row images. The second image acquisition system was performed with a Raspberry pi native camera module 
(Cam2). It was used for capturing intra row images. Cam2 is supported in the latest version of Raspbian, Raspberry Pi's preferred operating system. And to avoid the interference of shadows and other illumination related obstacles Cam2 fixed a special light ring (Magideal LuxurySelfie Luminous LED Light Up Phone Ring). In [2] the shot system with a high-speed camera coupled with backlighting LED was used in precision spraying for different surface orientations. The means to reduce the amount of herbicide applied by directing droplets more accurately towards the weeds was investigated. In [3] an automated sensing system was developed for spotapplication in blueberry fields. The system included four digital cameras. In [4] a proximal spectral-reflectancebased plant discrimination sensor used a new stabilized three-wave length laser diode module for plant illumination was developed. The novel sensor can sequentially emit identically polarized laser light beams through a common aperture, along one optical path. Each laser beam entered a multi-spot beam generator produced 15 parallel, collimated laser beams spaced over a 230$\mathrm{mm}$ span. The outdoor performance of the wo plants was experimentally assessed by the sensor. Plant discrimination was based on calculating two different normalised difference vegetation indices. In [5] objectives were investigated using true colour (RGB) and colour-infrared (CIR) cameras under different ambient lighting conditions. The camera system is mounted on UAV. The system based on UAV imagery had the same ability to quantify crop responses to experimental treatments as ground-based recordings with cameras. But one of shortcomings that need to be taken into consideration is ambient light fluctuations. In [6] Two different cameras systems were employed for capturing images for network sets built. Some images were taken under controlled conditions with regard to camera stabilisation and illumination. Some images were shot with hand-held mobile phones in fields with changing lighting conditions and different soil types. The optimization of these shooting systems can in some degree improve the segmentation error caused by the effects of light. However, the effect of light on segmentation can't be completely solved by these methods. In [7] a module for a weed removal mobile robot with camera obscura (Latin for "dark room") for lighting controlled conditions was developed.

Secondly some researchers classified the different lighting environments during image collection. And images under different light environments are collected for data processing. In [8] the images of the initial growth stages of crop and weed plants in maize fields were obtained under different light environment((a) a sunny day with high level of illumination with a certain tendency to image saturation, (b) a cloudy day where the illumination is considerably reduced compared to (a), (c) a day with intermediate illumination levels with respect the two former) for detecting curved and straight crop rows. The study found that for the classical excess green vegetation index $(E x G)$ robust enough against changes in three light environments in maize fields. The experiments showed that when the images were shot with fixed exposure time and aperture in three light environments in maize fields, the image segmentation results and consequently the final results were not affected. In [6] six different data sets were obtained which have variations with respect to lighting, resolution, and soil type for the convolutional neural network training. In [9] under three different illumination conditions field experiments were conducted. The results under these three different illumination conditions showed that the accurate identification rates on lettuce, cauliflower and maize were all above $95 \%$.

Thirdly Some researchers tried use new algorithm replacing traditional threshold segmentation method. In [2] an instance-based learning approach (a kappa-Nearest Neighbors (kappa-NN) algorithm) for segmentation of crop images was able to automatically discriminate the green textures (crop and weeds) from the rest of the ground under different outdoor conditions, namely light conditions and stages of crop growth. And the method proposed provided good results for all the different conditions including burned and saturated images. In [6] the robust algorithm vision-based in an agricultural environment was proposed for autonomous navigation of field robots. The method is based on a Particle Filter (PF) using a novel measurement model. And a model image from the particle was constructed. It was compared directly with the measurement image after elementary processing, such as down-sampling, excessive-green filtering and thresholding. The experiments showed that algorithm in several maize fields with different row patterns, varying plant sizes and diverse lighting conditions was demonstrated.

Finally, some researchers extract a lot different color features of field images under different lighting conditions for data analysis. In [12] considering the adverse effect of illumination variation on image processing, the HIS (hue, saturation, intensity) color model was used to process images. The threshold algorithm based on the $\mathrm{H}$ component was used to produce gray scale image. The experimental results were conducted that the guidance system successfully adapted to changes in natural light. In [9] a modified excess green feature ( $\mathrm{g}-\mathrm{r}>\mathrm{T} \& \mathrm{~g}-\mathrm{b}>\mathrm{T}$ ) was used to segment plant material from back ground in color images within the normalized red, green and blue chromatic coordinates (rgb). To cope with variable natural light the threshold $\mathrm{T}$ was automatically selected by the maximum variance (OTSU) algorithm. Field experiments were conducted under three different illumination conditions. The results demonstrated that the accurate identification rates on lettuce, cauliflower and maize were all above 95\%. The positional measurement error was within $+/-15 \mathrm{~mm}$. In [3] the color feature of ratio of $255^{*} \mathrm{G} /(\mathrm{R}+\mathrm{G}+\mathrm{B})$ was used that Red-Green-Blue (RGB) images from the automated sensing system were converted to normalized green ratio binary images. The segmentation for the discrimination in an automated sensing system for spot-application of herbicide in pruned wild blueberry fields. The robust and effective algorithm was developed for Green contrast between weed and non-weed area in pruned wild blueberry fields. The experiments showed the normalized green ratio algorithm showed very reliable accuracy under different outdoor light conditions (>500 lux) and 
shade conditions. In [5] four different vegetation indices (ExG, NGRDI, NDVI and ENDVI) were used for segmentation under different ambient lighting conditions. The study showed that there were shortcomings that need to be taken into consideration: (1) angular variation in reflectance (bidirectional reflectance), (2) stitching and (3) ambient light fluctuations. The study showed that to avoid the effects of light the special segmentation features which were proposed in the literature were effective for the specific crops at different growth stages.

\subsection{The effects of light on weed identification algorithm}

The weed identification means to distinguish weeds and crops. Since the data source of weed identification algorithm based visual is image and the quality of image is affected by light, a lot of experts have done lots research on the influence of light on weed identification. In order to improve identification results more effective methods are to improve the identification features and try new algorithm.

The following literatures are mainly about the attempt of new identification algorithm. In [1] a fuzzy real time classifier on leaf textures was used for a weed detecting robotic model in sugarcane fields. The designed robotic prototype correctly identified the sugarcane crop among nine different weed species. The system detected weeds with $92.9 \%$ accuracy. In [6] a convolutional neural network was used for weed identification. The method was capable of recognising plant species respect to different lighting, resolution, and soil type. The experiment showed the method for those 22 species was able to achieve a classification accuracy of $86.2 \%$. In [7] lighting and sharpness problems during acquisition step were proposed in weed detection in vegetable crops using outdoor images. A useful algorithm was developed using image filtering to extract color and area features for weed identification. In [13] a new training procedure with noisy patterns for ensembles of neural networks was introduced. The new noisy ensembles were applied to images from orange groves to determine where weeds were located using the proposed two-stage procedure. The results showed that the proposed system was suitable for weed detection in orange, and similar, groves.

The following literature suggests more identification features. In [14] to avoid the influence of different illumination on images, the color model was adopted and component to gray images was proposed. Modified Weeds Infestation Rate (MWIR) was used to realize realtime decision through the minimum error ratio of Bayesian decision under normal distribution. In [15] the feature set in sugar beet fields under natural, outdoor conditions was proposed which were made up of 14 indices feature. Among all the features, excess green $(\mathrm{ExG})$, green minus blue $(\mathrm{GB})$ and color index for vegetation extraction (CIVE) offered the highest average accuracy. And the PCA analysis was used. The feature set was reduced to four important indices. A total of 474 field images of sugar beet and thistles were collected and divided into six different groups based on illumination, scale and age. But the classification accuracy of four important indices combinations was similar to that obtained by only combining ExG and GB which was around $95 \%$. The validation experiments showed that sunlight followed by the size of the plant, which was related to its growth stage, were the two most important factors affecting the classification.

\section{Noteworthy issues}

Accurate identification of weeds is the basis for automatic weed removal. At present, the main treatments for the impact of natural light on the original image are to introduce controllable light environment, to find the algorithm and its internal parameters with good robustness to changed illumination environment, and to establish the basic data sets of various light conditions of weeds and crops. But the current research is mostly indoor controlled light environment, and the outdoor research is relatively rough.

Sheltering from sunlight in outdoor field is a feasible approach. And the experimental study of quantization of illumination conditions under sheltering from sunlight in outdoor field will be more helpful to get the relationship between illumination and recognition results.

Due to Chemical properties of the crops and weeds at different growth stages show different photosensitive nature the collected images in the field have different quality. The relationship between them can be analyzed through further basic experimental research. It will be done under the quantitative indexes of different sheltering from sunlight, different supplementary light sources and different light intensity in outdoor field.

\section{Conclusion}

The effects of light on the two main steps of green vegetation identification and weed recognition are reviewed in this paper. At present the development of the new capturing system, different image collection under the different lighting environments, new algorithm used replacing traditional threshold segmentation method and a lot new color features of field images extracted under different lighting conditions are the main methods for solving the effects of light on green vegetation identification. The new identification features and new algorithm may be more effective methods to improve identification results for weed identification under different light environment. In the development of the new capturing system methods are suggested for possible solutions to the processing parameters of weed identification under the influence of light with sheltering from sunlight in outdoor field constructing controllable outdoor light environment and quantifying the experimental parameters, so as to help determine a more accurate weed identification scheme for different weeds in different crops growth stages. This will provide a reference for subsequent relevant studies. 


\section{Acknowledgments}

This work has received funding from Regional Science Foundation of National Natural Science Foundation of China "The research on feature extraction of vegetable community in weed identification" (61563007).

\section{References}

1. M Sujaritha. S Annadurai. J Satheeshkumar. et al., Computers and Electronics in Agriculture, 134, pp.160-171 (2017).

2. M Mathieu, B Hassina, L Frederic. PRECISION AGRICULTURE.15, pp.241-254 (2014).

3. YK Chang, Q Zaman, T Esau, AW Schumann. Applied Engineering in Agriculture, 30, pp.143-152 (2014).

4. S Askraba, A Paap, K Alameh, J Rowe, C Miller. IEEE SENSORS JOURNAL. 16, pp.6680-6686 (2016).

5. J Rasmussen, G Ntakos, J Nielsen, J Svensgaard, RN Poulsen, S Christensen, EUROPEAN JOURNAL OF AGRONOMY, 74, pp.75-92 (2016).

6. M Dyrmann, H Karstoft, S Midtiby Henrik. Biosystems Engineering, 151, pp.72-80 (2016).
7. PR Camilo Andrés, MV Manuel Alejandro, SG Leonardo Enrique, Revista Facultad de Ingeniería Universidad de Antioquia, 80, pp.124-130 (2016).

8. ID García-Santillán, M Montalvo, JM Guerrero, G Pajares. , Biosystems Engineering, 156, pp.61-79 (2017).

9. L Nan, Z Chunlong, C Ziwen, et al., International Journal of Agricultural and Biological Engineering, 8, pp.20-29 (2015).

10. J Arroyo, G Pajares. Computers and Electronics in Agriculture, 127, pp.669-679 (2016).

11. S Hiremath, FKV Evert, CT Braak, A Stein, GVD Heijden. Biosystems Engineering, 121, pp.85-95 (2014).

12. Q Meng ,R Qiu, J He, M Zhang, X Ma. Computers and Electronics in Agriculture, 112, pp.128-138 (2015).

13. TS Joaquin, N Patricio, Biosystems Engineering, 123, pp.40-55 (2014).

14. $\mathrm{T}$ Jing-Lei, $\mathrm{C}$ Xiao-Qian, $\mathrm{M}$ Rong-Hui, et al., Computers and Electronics in Agriculture, 122, pp.103-111 (2016).

15. K Wajahat, GR Francisco Jose, $\mathrm{N}$ Jon, et al., Computers and Electronics in Agriculture, 112, pp.10-19 (2015). 